\title{
Supersonic Pitch Damping Predictions of Blunt Entry Vehicles from Static CFD Solutions
}

\author{
Mark Schoenenberger* \\ NASA Langley Research Center, Hampton Virginia 23681
}

\begin{abstract}
A technique for predicting the supersonic pitch damping of blunt axisymmetric bodies from static CFD data is presented. The contributions to static pitching moment due to forebody and aftbody pressure distributions are broken out and considered separately. The one-dimension pitching moment equation is cast to model the separate contributions from forebody and aftbody pressures with no traditional damping term included. The aftbody contribution to pitching moment is lagged by a phase angle of the natural oscillation period. This lag has been predicted by others using computational methods; it represents the time for aftbody wake structures to equilibrate while the body is oscillating. For a case with constant forebody and aftbody pitch-moment slopes, the characteristic equation of this formulation indicates that the lagged backshell moment adds a damping moment equivalent in form to a constant pitch damping term. The nonlinear moment equation is then linearized to yield an expression for pitch damping at a local angleof-attack that is a function of the local contribution to pitching moment by the backshell and a lag time. CFD calculations of a capsule backshell's contribution to the static pitching moment for a range of anglesof-attack is used to predict pitch damping coefficients. These predictions are compared with ballistic range data taken of the Mars Exploration Rover (MER) capsule and forced oscillation data of the Mars Viking capsule. The lag model appears to capture dynamic stability variation due to backshell geometry and Mach number. This lagged backshell pitching moment method can be used to make preliminary estimates of the dynamic stability of a blunt body traveling at supersonic speeds.
\end{abstract}

\section{Nomenclature}

$\begin{array}{llll}a & \text { Speed of sound } & S & \text { Reference area } \\ B & \text { Linearized solution constant } & t & \text { Lag time } \\ C_{m_{\alpha}} & \text { Pitching moment coefficient } & t_{\phi} & \text { Lag time } \\ C_{m_{\alpha(B S)}} & \text { Backshell contribution to total } C_{m_{\alpha}} & V & \text { Velocity } \\ C_{m_{\alpha(F B)}} & \text { Forebody contribution to total } C_{m_{\alpha}} & X & \text { Arbitrary equation variable } \\ C_{m_{\dot{\alpha}}} & \text { Pitch damping coefficient }\left(\frac{\partial C_{m}}{\partial \frac{q d}{2 V}}\right) & \text { Greek } & \\ C_{m_{o}} & \text { Linearized } C_{m} \text { offset } & \alpha & \text { Angle of attack } \\ d & \text { Diameter } & \alpha_{o} & \text { Linearization angle of attack } \\ E & \text { Linearized solution constant } & \gamma & \text { Linearized harmonic oscillator offset } \\ f & \text { Frequency (cycles per second) } & \epsilon & \text { Angle of attack deviation from } \alpha_{o} \\ I_{y y} & \text { Moment of inertia about pitch axis } & \lambda & \text { Characteristic equation roots } \\ M_{B S} & \text { Dimensional pitching moment (backshell) } & \xi & \text { Harmonic oscillator damping coefficient } \\ q & \text { Dynamic pressure } & \rho & \text { Flowfield density }\end{array}$

\footnotetext{
*Aerospace Engineer, Senior Member AIAA
} 


Greek (cont'd)
$\phi$
$\omega$
$\omega_{o}$
$\omega_{1}$
$\omega_{2}$

Greek (cont'd)

Wake lag phase angle

Harmonic oscillator natural frequency $(\mathrm{rad} / \mathrm{s}$ )

Harmonic oscillator spring constant

Forebody spring constant

Backshell spring constant
Subscripts

$\infty$

Overscript

$\cdot$

$\cdot \cdot$
Freestream

1st time derivative

2nd time derivative

\section{Introduction}

The dynamic stability of blunt bodies is an important area of research for planetary entry mission design. As an entry capsule descends to low supersonic speeds (Mach_ $\approx 3.0$ to 4.0 and below typically), base pressure contributions to the pitching moment become significant and can result in dynamic instabilities. These instabilities cause oscillations in angle-of-attack to increase in amplitude, potentially creating problems for parachute deployment and in extreme cases can cause the vehicle to tumble. It is therefore desirable to characterize the pitch damping of a blunt entry vehicle as well as possible in the low supersonic regime to mitigate any flight performance risks through entry trajectory modifications or stabilization devices like a supersonic drogue parachute.

There are several test methods available to characterize the pitch damping of blunt vehicles. Forced oscillations tests were used extensively for the Mars Viking missions, ${ }^{1}$ Apollo and other vehicles. Forced oscillation tests offer several attractive features. Highr rate, accurate damping measurements can be made, and tunnel conditions and freestream gases are both controllable. Sting effects, however, raise the questions of whether the data is valid for a free flying capsule as the wake structure is thought to be the dominant source of dynamic instability. Some studies ${ }^{2,3}$ have shown that sting effects can be mitigated to some degree, but they remain a source of error. Ballistic range testing is another attractive option for blunt vehicles. This type of testing has the benefit of a free flying model with no sting effects. In some facilities, ${ }^{4}$ the freestream pressure and gas (air, $\mathrm{CO}_{2}$, etc.) can be controlled as well. However, extracting dynamic derivatives via ballistic range testing is a complicated and less definitive process than reducing forced oscillation data. Pitch damping is extracted through parameter identification techniques. The aerodynamic coefficients are determined by fitting simulated trajectories through time, position, and attitude data collected at discrete points as the model flies down the range. The constants in assumed functional forms describing the aero coefficients are varied to minimize the error between the simulated trajectory and shadowgraph data points. In such tests, Mach number and oscillation amplitude are varying rapidly as the model decelerates. With these variables changing significantly during each shot, and considering the relatively sparse data (up to 50 photo stations), pitch damping cannot be resolved to the precision that forced oscillation data can achieve. Both of these techniques are time consuming and expensive. Numerical determination of pitch damping by way of time-accurate computation fluid dynamics (CFD) is a relatively new option and to date has not been validated. The ability to quickly and accurately determine the general pitch damping characteristics of a given blunt body would be a great benefit to entry aerodynamicists. It would allow more effective preliminary mission planning without expensive experimental programs, and would help reduce the scope of such experimental programs when higher fidelity is required. While not validated, some significant advances have been made with CFD that have shed some light on the physics of blunt body dynamic stability.

Teramoto et $\mathrm{al}^{5}$ have shown evidence that the physics driving the dynamic instability of a blunt entry vehicle is a lag of the base pressure distribution behind its instantaneous attitude as the body undergoes oscillatory motion. They show computational results where the annular vortex in the wake flow behind an entry capsule takes a finite amount of time to reach a steady state structure when the body attitude is changed. For oscillatory motion, the wake structure lags by this amount of time behind the current body attitude. For the case they presented, this lag results in a dynamic instability (neglecting the forebody damping). This idea of a lagging base pressure distribution is taken from this computational work and applied to available static CFD solutions in an attempt to predict the dynamic stability characteristics of the Mars Exploration Rover and Viking entry capsules. It was hypothesized that static measurements or CFD predictions of backshell contributions to static stability might be an indicator of how dynamically stable or unstable a configuration might be. This work shows that lagging the backshell static moments does predict damping behavior that is consistent with experimental data. 


\section{Analysis}

Consider the blunt body in Figure 1 traveling at a constant velocity, $V_{\infty}$. The body is free to oscillate and is statically stable. This configuration could represent a free-to-oscillate wind tunnel test where the tunnel velocity is constant and the model is constrained to rotate only about the sting axis. The pressure distribution on the forebody dominates the static aerodynamic forces and moments. The forebody also provides positive damping when oscillating, but the separated wake flow can introduce dynamic instabilities at small angles. As described earlier, it is believed that a lag of the backshell pressure distribution caused by the inertia of the separated wake structure is the mechanism that produces the instability. That phenomena is what will be modeled here.

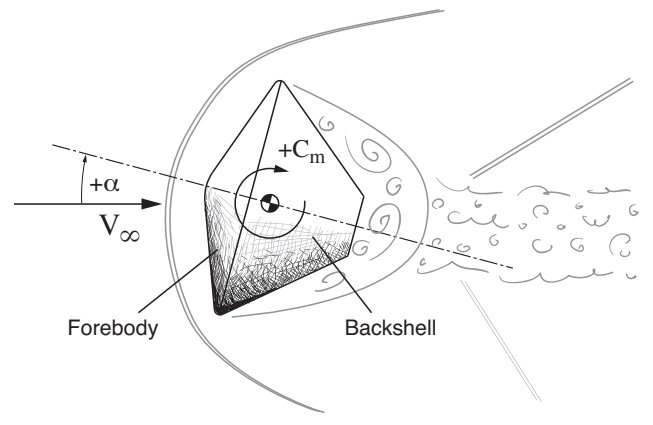

Figure 1. Blunt capsule traveling constant supersonic velocity, free to oscillate

The equation of motion for this configuration is that for a simple harmonic oscillator and textbooks readily provide solutions for the angle of attack history of the body for constant coefficients. Other solutions exist for conditions where a body is free to heave due to lift and free to decelerate due to the drag force acting on the vehicle. ${ }^{6}$ The special case of constant velocity with no heaving due to lift is used here to predict the pitch damping coefficient from static aerodynamics. A setup with more degrees of freedom would not change the contribution to amplitude growth from the pitch damping term, but additional degrees of freedom would add terms to the equations that are irrelevant to this analysis. The pitch damping predicted here should be relevant to other flight conditions with additional degrees of freedom.

\section{Linear Analysis}

The 1-D pitching moment equation for a blunt body constrained to constant velocity and a specified center of rotation (typically for a wind tunnel test, this is at or near an expected center of gravity) is given below. Summing the moments yields:

$$
I_{y y} \ddot{\alpha}-\frac{1}{2} \rho V_{\infty}^{2} S d C_{m_{\dot{\alpha}}} \frac{d}{2 V_{\infty}} \dot{\alpha}-\frac{1}{2} \rho V_{\infty}^{2} S d C_{m_{\alpha}} \alpha=0
$$

Which can be re-expressed in the form:

$$
\ddot{\alpha}+2 \xi \dot{\alpha}+\omega_{o}^{2} \alpha=0
$$

where

$$
\xi=-\frac{C_{m_{\dot{\alpha}}} q S d^{2}}{4 I_{y y} V_{\infty}}
$$

and

$$
\omega_{o}^{2}=-\frac{C_{m_{\alpha}} q S d}{I_{y y}}
$$

From the classic solution of a harmonic oscillator, substituting 


$$
\alpha=e^{\lambda t}
$$

into equation 2 yields the 2 nd order characteristic equation

$$
\left(\lambda^{2}+2 \xi \lambda+\omega_{o}^{2}\right) e^{\lambda t}=0 \Rightarrow \lambda^{2}+2 \xi \lambda+\omega_{o}^{2}=0
$$

The roots of equation 6 provide the solution for equation 2 when substituted back into equation 5 . Here, the forebody contribution to pitch damping is neglected by setting the usual damping term, $\xi$, to zero in Equation 2. This is not typically a valid assumption, but is done here to more clearly illustrate the effect of lagging the backshell contribution to the static pitching moment. A nonzero value for $\xi$ to account for the forebody damping will be incorporated into the solution developed in this section later in the paper to estimate the total damping of some example configurations.

Now the pitching moment slope is separated into forebody and backshell contributions with the assumption that both are constant.

$$
C_{m_{\alpha}}=C_{m_{\alpha(F B)}}+C_{m_{\alpha(B S)}}
$$

By imposing a lag in the backshell contribution to pitching moment as described by Teramoto et al, the moment equation can be cast in the following manner

$$
\ddot{\alpha}(t)+\omega_{2}^{2} \alpha\left(t-t_{\phi}\right)+\omega_{1}^{2} \alpha(t)=0
$$

Where

$$
\omega_{2}^{2}=-\frac{C_{m_{\alpha(B S)}} q S d}{I_{y y}}
$$

and

$$
\omega_{1}^{2}=-\frac{C_{m_{\alpha(F B)}} q S d}{I_{y y}}
$$

Again we have the exponential form of the solution given in Equation 5. For the backshell term we introduce the lag time into the assumed form of the angle of attack

$$
\alpha\left(t-t_{\phi}\right)=e^{\lambda\left(t-t_{\phi}\right)}=e^{\lambda t} e^{-\lambda t_{\phi}}
$$

Substituting Equations 5 and 11 into Equation 8 yields

$$
\left(\lambda^{2}+\omega_{2}^{2} e^{-\lambda t_{\phi}}+\omega_{1}^{2}\right) e^{\lambda t}=0 \Rightarrow \lambda^{2}+\omega_{2}^{2} e^{-\lambda t_{\phi}}+\omega_{1}^{2}=0
$$

The exponent in the lagged pitching moment term in Equation 12 can be expressed as a truncated Maclaurin series. Recall the series expansion:

$$
e^{X}=1+X+\frac{X^{2}}{2 !}+\frac{X^{3}}{3 !}+\ldots
$$

For small values of $\lambda t_{\phi}$ the first two terms of the expansion are a reasonable approximation. Substituting into Equation 12 yields

$$
\lambda^{2}+\omega_{2}^{2}\left(1-\lambda t_{\phi}\right)+\omega_{1}^{2}=0
$$

Equations 4, 7, 9 and 10 can be used to combine the $\omega_{2}$ and $\omega_{1}$ terms. Rearranging we have

$$
\lambda^{2}-\omega_{2}^{2} t_{\phi} \lambda+\omega_{o}^{2}=0
$$

Equation 14 is now in the exact form of Equation 6. This shows that with the few approximations and assumptions made, a lag of the pressure distribution on the backshell of a blunt vehicle creates a damping behavior. Comparing the second terms in Equations 6 and 14, a relation for pitch damping can be determined. 


$$
2 \xi=-\frac{C_{m_{\dot{\alpha}}} q S d^{2}}{2 I_{y y} V_{\infty}}=-\omega_{2}^{2} t_{\phi}=\frac{C_{m_{\alpha(B S)}} q S d}{I_{y y}} t_{\phi}
$$

The pitch damping can then be determined from the base contribution to the static pitching moment and a lag time.

$$
C_{m_{\dot{\alpha}}}=-\frac{2 C_{m_{\alpha(B S)}} V_{\infty} t_{\phi}}{d}
$$

With some manipulation, we can get a more physical sense of the parameters influencing the damping effects due to the backshell. First, the lag time will be expressed as a phase angle or fraction of the period of one oscillation cycle. The roots of Equation 14 include the natural frequency of oscillation. These roots are:

$$
\lambda_{1,2}=-\xi \pm i \omega
$$

where the natural frequency, $\omega$, in $\mathrm{rad} / \mathrm{s}$ is

$$
\omega=\sqrt{\omega_{o}^{2}-\xi^{2}}=\sqrt{-\left(\frac{C_{m_{\alpha}} q S d}{I_{y y}}\right)-\left(\frac{2 C_{m_{\alpha(B S)}} q S d}{I_{y y}} t_{\phi}\right)^{2}}
$$

The $C_{m_{\alpha}}$ term dominates Equation 16 for our cases where $t_{\phi}$ is small and $C_{m_{\alpha(B S)}}$ is small compared to the total pitching moment slope. Therefore, the oscillation frequency is roughly the same as the undamped value, $\omega \approx \sqrt{-C_{m_{\alpha}} q S d / I_{y y}}$. Now, the lag time can be expressed as a fraction of a complete cycle of oscillation. Recall the conversion of frequency in radians per second to cycles per second:

$$
\begin{gathered}
f=\frac{\omega}{2 \pi} \\
t_{\phi}=\left(\frac{\phi}{2 \pi}\right) \frac{1}{f}=\frac{\phi}{\omega}
\end{gathered}
$$

Substituting Equation 17 into Equation 15 yields a simple expression for the upper bound of the pitch damping due to the backshell pressure distribution lagging behind the capsule attitude:

$$
C_{m_{\dot{\alpha}}}=-\frac{2 C_{m_{\alpha(B S)}} V_{\infty} \phi}{d \omega}=-\frac{2 C_{m_{\alpha(B S)}}}{\sqrt{-C_{m_{\alpha}}}} \sqrt{\frac{2 I_{y y}}{\rho_{\infty} S d^{3}}} \cdot \phi
$$

This expression requires only some reference parameters specific to the vehicle, static aerodynamic data and a phase angle to determine the pitch damping at a given atmospheric condition (freestream density). This equation for the pitch damping due to backshell effects, when substituted into Equation 3, yields the following expression for the moment due to damping (second term in Equation 2):

$$
\frac{M_{B S-\text { damping }}}{I_{y y}}=2 \xi \dot{\alpha}=-C_{m_{\alpha(B S)}} \phi \sqrt{-\frac{q S d}{C_{m_{\alpha}} I_{y y}}} \dot{\alpha}
$$

This equation shows that the damping due to the backshell is directly proportional to both the pitching moment slope of the backshell as well as the phase angle of the pressure distribution lag time. The damping is also related to the square root of the geometric parameters of the capsule (area, diameter) as well as the dynamic pressure at which the vehicle is flying and inversely proportional to the square root of the static stability of the forebody and moment of inertia.

To predict the pitch damping for a particular configuration, static CFD solutions of a capsule along with its external dimensions and mass properties are all that are required except for one remaining item, the lag phase angle. Estimation of the lag time will be addressed below in the context of an example case. 


\section{Nonlinear $C_{m(B S)}$ Analysis}

The analysis shown thus far assumes that $C_{m_{\alpha(B S)}}$ is constant. For the wake contribution to $C_{m}$ for a blunt body, this may only be appropriate at small angles-of-attack. Linearizing Equation 8 about an angle-of-attack will allow predictions of pitch damping to be made in nonlinear regions.

First make the substitution:

$$
\alpha=\alpha_{o}+\epsilon
$$

It follows that

$$
\begin{aligned}
& \dot{\alpha}=\dot{\epsilon} \\
& \ddot{\alpha}=\ddot{\epsilon}
\end{aligned}
$$

Where $\alpha_{o}$ is the angle-of-attack about which the moment equation is linearized and $\epsilon$ is a small change in angle-of-attack from that point. For small deviations from the local angle-of-attack, the backshell pitching moment can be approximated by a linear curve:

$$
C_{m(b s)} \approx C_{m_{\alpha(b s)}} \alpha+C_{m_{o}}=C_{m_{\alpha(b s)}} \epsilon+C_{m_{\alpha(b s)}} \alpha_{o}+C_{m_{o}}
$$

The forebody contribution to pitching moment is still assumed to be linear. Substituting the linearized version of angle of attack and the linear approximation for the nonlinear backshell pitching moment into Equation 1, yields the following moment equation:

$$
I_{y y} \ddot{\epsilon}-\frac{1}{2} \rho V_{\infty}^{2} S d C_{m_{\dot{\alpha}}} \frac{d}{2 V_{\infty}} \dot{\epsilon}-\frac{1}{2} \rho V_{\infty}^{2} S d\left[\left(C_{m_{\alpha(F B)}}+C_{\left.m_{\alpha(B S)}\right)}\right)+C_{m_{\alpha}} \alpha_{o}+C_{m_{o}}\right]=0
$$

We again neglect the $C_{m_{\dot{\alpha}}}$ term here and introduce the lag time to the backshell contribution to static pitching moment. Now, the lagged pitching moment equation (Equation 8) expressed in terms of $\epsilon$ becomes

$$
\ddot{\epsilon}(t)-\frac{C_{m_{\alpha(b s)} q S d}}{I_{y y}} \epsilon\left(t-t_{\phi}\right)-\frac{C_{m_{\alpha(F B)}} q S d}{I_{y y}} \epsilon(t)=\frac{C_{m_{\alpha}} q S d}{I_{y y}} \alpha_{o}+\frac{C_{m_{o}} q S d}{I_{y y}}
$$

which can also be expressed as

$$
\ddot{\epsilon}(t)+\omega_{1}^{2} \epsilon(t)+\omega_{2}^{2} \epsilon\left(t-t_{\phi}\right)+\omega_{o}^{2} \alpha_{o}+\gamma=0
$$

where

$$
\gamma=-\frac{q S d}{I_{y y}} C_{m_{o}}
$$

Like the linear version, $\omega_{1}$ is the forebody pitching moment slope and remains constant in this analysis for all angles-of-attack. The form of Equation 22 looks similar in form to Equation 8 except for two constant terms which come from the linearization approximation. With a slightly different substitution for $\epsilon$ we can recover the same characteristic equation.

Let $\epsilon$ be of the form

$$
\epsilon=e^{\lambda t}+B+E
$$

and the lagged backshell version of $\epsilon$ is

$$
\epsilon\left(t-t_{\phi}\right)=e^{\lambda t} e^{-\lambda t_{\phi}}+B+E
$$

Substituting $\epsilon$ in Equations 23 and 24 into Equation 22 yields

$$
\lambda^{2}-\left.\omega_{2}^{2}\right|_{\alpha_{o}} t_{\phi} \lambda+\left.\omega_{o}^{2}\right|_{\alpha_{o}}+\left.\omega_{o}^{2}\right|_{\alpha_{o}} B+\left.\omega_{o}^{2}\right|_{\alpha_{o}} E=\left.\omega_{o}^{2}\right|_{\alpha_{o}} \alpha_{o}+\gamma
$$

Now the constants $B$ and $E$ can be set to the following values 


$$
\begin{gathered}
B=-\alpha_{o} \\
E=\frac{\gamma}{\omega_{o}^{2}}=\frac{C_{m_{o}}}{C_{m_{\alpha}}}
\end{gathered}
$$

And Equation 25 reduces to

$$
\lambda^{2}-\omega_{2}\left(\alpha_{o}\right)^{2} t_{\phi} \lambda+\omega_{o}\left(\alpha_{o}\right)^{2}=0
$$

which is exactly the same as Equation 14 with the caveat that the constants are valid only near the angle of attack about which the equation was linearized. Again, the second term in Equation 25 can be used to determine an expression for the pitch damping due to a lagged base pressure distribution.

$$
C_{m_{\dot{\alpha}}}\left(\alpha_{o}\right)=-\frac{2 \omega_{2}\left(\alpha_{o}\right)^{2} V_{\infty} I_{y y} t_{\phi}}{q S d}=-\frac{\left.2 C_{m_{\alpha(b s)}}\right|_{\alpha_{o}} V_{\infty} t_{\phi}}{d}
$$

Substituting the simplified expression for the natural frequency into Equation 27 as was done with solution to the linear moment equation yields similar expression for the pitch damping due to the lagged backshell pitching moment slope, independent of freestream velocity. Just like the linear expression, the local nonlinear dynamic stability is a function of freestream density, vehicle parameters, the backshell and forebody pitching moment slopes and a phase angle:

$$
C_{m_{\dot{\alpha}}}\left(\alpha_{o}\right)=-\frac{2 C_{m_{\alpha(B S)}} \mid \alpha_{o}}{\sqrt{-C_{m_{\alpha}}}} \sqrt{\frac{2 I_{y y}}{\rho_{\infty} S d^{3}}} \cdot \phi
$$

\section{Forebody damping}

For the complete description of the pitch damping of a blunt capsule, the forebody damping must be accounted for. This damping comes from differential changes to the pressure distribution over the forebody as the capsule oscillates (advancing portions see increased pressure and retreating portions see decreased pressure) and does not rely on any lag effects. Tobak and Wehrend ${ }^{7}$ use a Newtonian method to estimate this damping for arbitrary shapes in the hypersonic flow regime.

Adding the pitch damping term (recall $\xi$ as defined in Equation 3) back into Equation 25, we see that the forebody pitch damping can simply be added to the backshell contribution to arrive at the complete pitch damping of a capsule:

$$
\lambda^{2}+\left(2 \xi-\left.\omega_{2}^{2}\right|_{\alpha_{o}} t_{\phi}\right) \lambda+\left.\omega_{o}\right|_{\alpha_{o}}=0
$$

Therefore

$$
C_{m_{\dot{\alpha}(\text { total })}}=C_{m_{\dot{\alpha}(\text { Mod.Newtonian })}}-\frac{2 C_{m_{\alpha(B S)}} \mid \alpha_{o} V_{\infty} t_{\phi}}{d}
$$

\section{Test Cases}

The mathmatical model presented will now be applied to a real case where static CFD calculations are used to "predict" the pitch damping behavior of two different blunt body shapes, the Viking and Mars Exploration Rover (MER) entry capsules. The results will be compared to pitch damping data obtained experimentally for these two shapes. Two different methods were used to obtain the pitch damping data, ballistic range for MER, and forced oscillation in a wind tunnel for Viking. Pitch damping data of both vehicles using both experimenal techniques would be more ideal for these comparisons, but the objectives of these comparisons are for general agreement only, not definitive validation of the approach.

Figure 2 shows the dimensions of the Viking and MER outer mold lines (OML), each normalized by their maximum diameters. This figure shows the two backhells to be the primary difference between the outer 


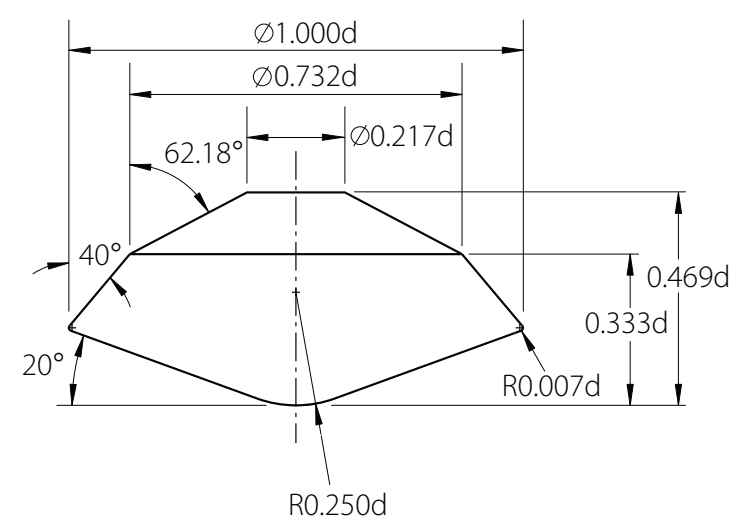

Viking

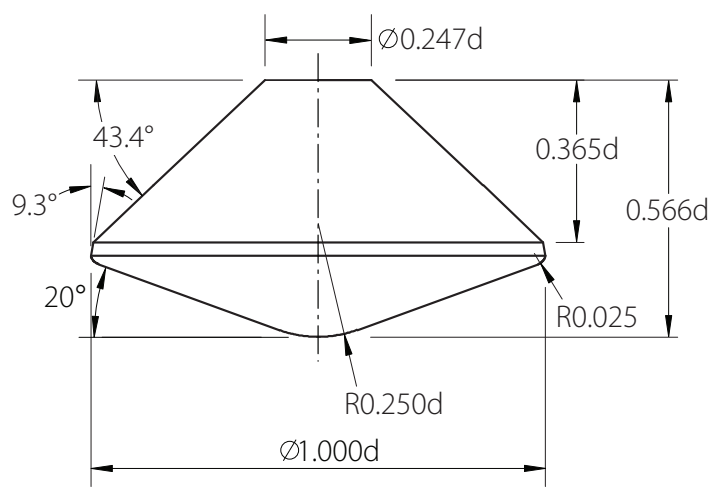

MER

Figure 2. Dimensions of Viking and MER capsules (nondimensionalized)

mold lines. This difference and the existence of experimental data at similar conditions are the reason for their selection.

\section{MER Ballistic Range Data}

An example case will be used to perform a quantitative analysis. The example that will be used is a ballistic range shot of the MER entry capsule conducted at the Aeroballistic Research Facility at Eglin Air Force Base. ${ }^{8,9}$ This case is used because it is relevant to this analysis, the dimensions and physical characteristics of the test models are well known, and pitch damping data were extracted from these tests with which comparisons may be made. The models tested were made of combinations of steel, tungsten and aluminum. The materials and proportions of each were varied to provide different center-of-gravity positions. They were fired down a 207 meter hallway instrumented with 50 shadowgraph stations that recorded their position and orientation. The time that each shadowgraph was taken was automatically recorded. This time and position information was then used in nonlinear parameter identification analysis to extract the static and dynamic aerodynamic coefficients.

Details of the test facilities and the various shot conditions are discussed in in an earliar paper by the author. ${ }^{9}$ The data reduction methodologies are described in more detail in a paper by Chapman et al. ${ }^{10}$ The relevant dimensions, mass properties and flow conditions from those tests will be provided here along with results relating to the static CFD solutions calculated for this analysis. Table 1 lists the model dimensions, moment of inertia, freestream density, and approximate freestream speed of sound for the Eglin tests. The Eglin ballistic range facility is open to the atmosphere and therefore the speed of sound and air density varies slightly from day to day. These representative values are sufficient for this analysis.

Table 1. Reference parameters for Eglin ballistic range and CFD predictions

\begin{tabular}{cc}
\hline Parameter & Ballistic Range Value \\
\hline \hline$d(m)$ & 0.07 \\
$S\left(m^{2}\right)$ & 0.00385 \\
$I_{y y}\left(k g-m^{2}\right)$ & $1.55 E-04$ \\
$\rho_{\infty}\left(\mathrm{kg} / \mathrm{m}^{3}\right)$ & 1.23 \\
$a(\mathrm{~m} / \mathrm{s})$ & 348 \\
\hline
\end{tabular}




\section{Viking Forced Oscillation Data}

During the Mars Viking project in the early to mid 1970's, extensive forced oscillation experimental data were obtained for the Viking aeroshell. ${ }^{1}$ A portion of these data will be used to compare to results of the present analysis using CFD solutions of the Viking capsule. For comparisons to the CFD solutions, only the pitch damping results will be used. Parameters from the MER ballistic range testing will be used to calculate $C_{m_{\dot{\alpha}}}$ values for comparison with these Viking data as well as the MER CFD predictions and ballistic range data. The use of one set of physical parameters and atmospheric conditions will simplify the comparisons of all these disparate data sources. The Viking project concluded that differences in the atmosphere in which the capsule is flying has little impact on the pitch damping of the vehicle. Viking also concluded that no corrections were required to account for damping data obtained from scaled vehicles to predict flight behavior. ${ }^{11}$ These conclusions were subsequently validated by flight data from two successful entries and landings of Viking capsules as well as the by the use of Viking data to predict the Mars Pathfinder entry behavior. ${ }^{12}$ Ideally, to compare the results of this pitch damping prediction method to experimental data of two different geometries, the validation data would be of equivalent fidelity. MER and Viking data were selected even though the pitch damping data derives from two different experimental techniques because the forebodies of those vehicles are nearly identical. Therefore any differences in damping should be due to the backshell contributions (and in this case, test technique). Therefore, for the level of accuracy sought for the present analysis, it will be assumed that pitch damping values determined by ballistic range or forced oscillation are of similar fidelity and insensitive to capsule size and atmospheric differences (air versus $\mathrm{CO}_{2}$ ). Again, the goal of the present analysis is to determine order-of-magnitude pitch damping characteristics and general trends driven by backshell geometry and vehicle velocity.

\section{LAURA Calculations}

Three angle-of-attack sweeps were calculated using the Langley Aerothermodynamic Upwind Relaxation Algorithm (LAURA). ${ }^{13}$ LAURA is a finite volume, shock capturing algorithm capable of calculating flows in chemical and thermal non-equilibrium. The solutions generated for this analysis are all laminar, perfect gas, thin-layer Navier stokes solutions. The molecular weight and ratio of specific heats used for the various solutions were appropriate to Mars entry $(43.26 \mathrm{~kg} / \mathrm{kmol}$ and approximately 1.3 respectively). The MER CFD cases were those used in the MER aerodynamic database ${ }^{14}$ and the flow conditions taken from the reference Mars trajectory used for those cases. Additional angles-of-attack were run to better resolve the backshell pitchign moment curve. The Viking CFD cases were run at very similar conditions with a grid of the Viking OML with similar fidelity to the MER grids. These solutions were used as they were readily available and relevant to available experimental data. The intent of this first look at CFD data was to determine if the static pitching moments on representative vehicles were of the correct magnitudes and slopes such that a reasonable lag time could produce dynamic instabilities similar to those observed in experiement.

Table 2 lists the solutions calculated for each $\alpha$-sweep. The pitching moment coefficients due to the backshell only were extracted from the pressure distribution over the surface cells of the backshell from each of these cases. The pitching moment coefficient curves determined from these solutions are plotted in Figure 3. Note that for each condition and shape there is an initial steep static stability near $\alpha=0^{\circ}$. The pitching moment decreases with $\alpha$ rapidly and reaches a minimum before shallowing and returning to almost no contribution at large angles. The location and magnitude of the most negative $C_{m(b s)}$ point is different for each configuration but the general character of the backshell moment contribution is similar.

Table 2. Angle-of-attack Sweep CFD Solutions

\begin{tabular}{cccccccccccc}
\hline Case & $2^{\circ}$ & $4^{\circ}$ & $6^{\circ}$ & $8^{\circ}$ & $11^{\circ}$ & $12^{\circ}$ & $14^{\circ}$ & $16^{\circ}$ & $18^{\circ}$ & $21^{\circ}$ & $26^{\circ}$ \\
\hline \hline $\operatorname{MER}(\mathrm{M}=2)$ & $\mathrm{x}$ & $\mathrm{x}$ & $\mathrm{x}$ & $\mathrm{x}$ & $\mathrm{x}$ & & $\mathrm{x}$ & $\mathrm{x}$ & $\mathrm{x}$ & $\mathrm{x}$ & $\mathrm{x}$ \\
$\operatorname{MER}(\mathrm{M}=3)$ & $\mathrm{x}$ & $\mathrm{x}$ & $\mathrm{x}$ & $\mathrm{x}$ & $\mathrm{x}$ & & $\mathrm{x}$ & $\mathrm{x}$ & & & \\
$\operatorname{Viking}(\mathrm{M}=3)$ & $\mathrm{x}$ & $\mathrm{x}$ & $\mathrm{x}$ & $\mathrm{x}$ & & $\mathrm{x}$ & $\mathrm{x}$ & $\mathrm{x}$ & & & \\
\hline
\end{tabular}




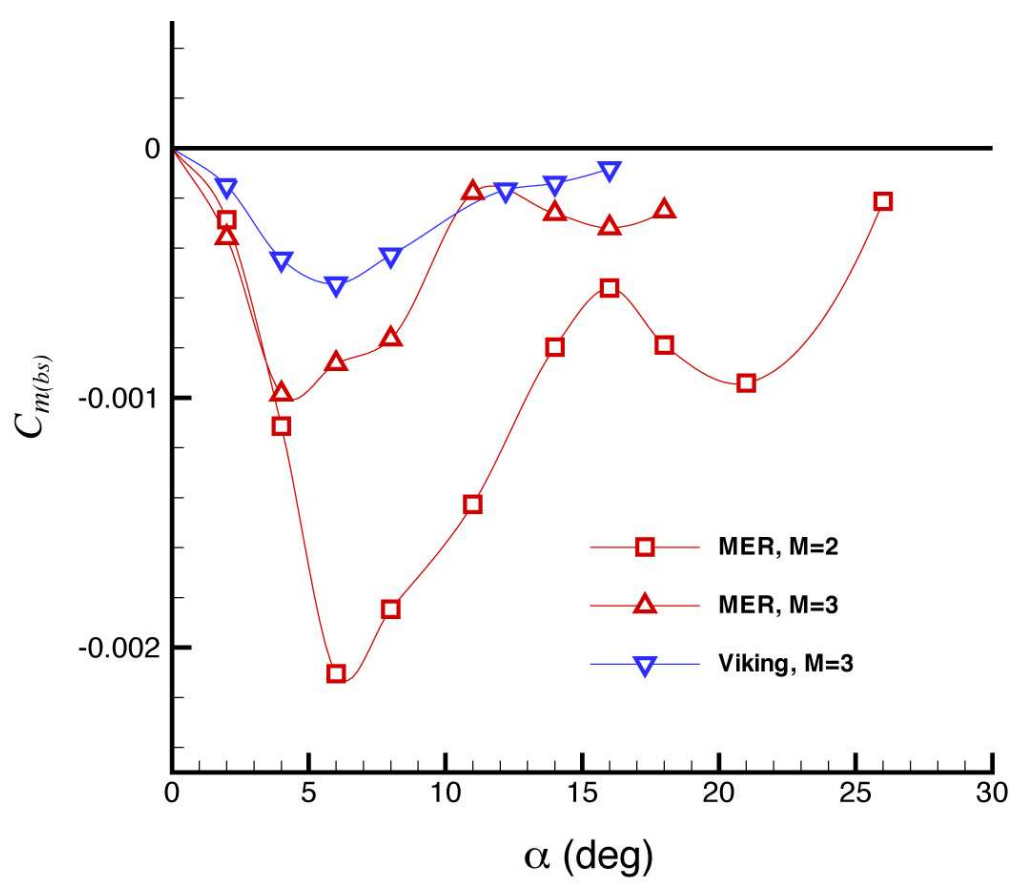

Figure 3. LAURA calculations of backshell contribution to pitching moment coefficient.

\section{Phase Angle Selection for Backshell Pitching Moment Lag Time}

The time lag of the base pressure distribution is a key component to this methodology. However, a rigorous basis upon which a lag time can be estimated does not exist. The proper understanding of any lag of the wake structure should be determined from experiment, looking for sensitivities to Reynolds number, oscillation frequency, Mach number and other parameters. Currently, there are some estimates available from unsteady CFD by Teramoto et al. ${ }^{\text {? }}$ The interpretation of the CFD data gives some theoretical understanding of what might drive the wake pressure lag. However, experimental work is necessary to validate and better understand the CFD predictions.

The Teramoto calculations showed that a $15^{\circ}$ phase angle corresponds to a lag time of 0.0021 seconds for a $100 \mathrm{~mm}$ diameter blunt capsule oscillating at $20 \mathrm{~Hz}$ (sinusoidal forced oscillation) at Mach=1.3. To demonstrate the pitch damping predicted by wake lag in this paper, the Teramoto phase angle $\left(\phi=15^{\circ}\right)$ will be used. To justify its use, let us look at the oscillation frequencies imposed or observed in the Teramoto unsteady CFD predictions, ballistic range, and forced oscillation tests. The Strouhal number will be used for this comparison. This nondimensional parameter is often used when scaling models for dynamic tests. The Strouhal number, defined in Equation 30, can be thought of as the number of oscillations that occur for a given distance traveled through the atmosphere (or amount of freestream air passing over a wind tunnel model).

$$
S t=\frac{\omega d}{2 V_{\infty}}
$$

The Strouhal numbers at the conditions where the lag prediction will be compared to MER experimental data are very similar to the value specified in the Teramoto CFD predictions: $S t_{\text {Teramoto }}=0.015$, $S t_{M E R, M=2}=0.016$ and $S t_{M E R, M=3}=0.011$. The Strouhal number at the Mach 3.0 Viking forced oscillation condition is roughly twice the Teramoto value: $S t_{V i k i n g, M=3}=0.032$. It is not clear what parameters the phase lag is sensitive to or to what degree, so the Teramoto value of $15^{\circ}$ will be used for all comparisons. It is reasonable to think that, all things being equal, a capsule oscillating more quickly (higher Strouhal number) would see the wake flow lag by a greater phase angle, but how phase angle varies with reduced frequency (and other parameters like Mach number) is beyond the scope of this paper. 
With the selection of a phase angle for these comparisons, we return now to Equations 27 and 28 . The local pitch damping coefficient can be calculated by multiplying the local pitching moment due to the backshell by single constant. For the MER ballistic rang conditions the expression for local pitch damping due to the lagged backshell static stability curve becomes:

$$
C_{m_{\dot{\alpha}}}=-\frac{2 C_{m_{\alpha(B S)}} \mid \alpha_{o} V_{\infty} t_{\phi}}{d}=-\frac{2 C_{m_{\alpha(B S)}} \mid \alpha_{o}}{\sqrt{-C_{m_{\alpha}}}} \sqrt{\frac{2 I_{y y}}{\rho_{\infty} S d^{3}}} \cdot \phi=-\left.24.1 \cdot C_{m_{\alpha(B S)}}\right|_{\alpha_{o}}
$$

\section{Forebody Pitch Damping Contribution}

For the complete dynamic stability of the MER and Viking capsules the contribution from forebody damping is required. The modified Newtonian method is extended to supersonic conditions for an approximate estimate of the forebody contribution to pitch damping as no analytical or empirical techniques have been developed for blunt supersonic flows. For a $C_{p, \max }$ of 1.87 and a center-of-gravity position of $x_{c g} / D=.2498$, the pitch damping of the MER forebody is approximately:

$$
C_{m_{\dot{\alpha}(\text { Mod.Newtonian })}}=-.316
$$

\section{Range of Applicability}

Before using the relations developed above to to generate pitch damping curves, it is important to estimate the valid range of angle-of-attack for which these relations apply. Figure 4 shows two Mach=3.0 LAURA CFD solutions used to generate the pitching moment curves of the MER capsule. The first plot, the MER capsule at $4^{\circ}$ angle-of-attack, shows a fully separated wake region with the separation streamline extending far from the backshell. The second plot in Figure 4 shows the MER capsule at $18^{\circ}$ angle-of-attack. This case shows the shear layer is very close to the backshell, on the windward side, if not completely attached. For such a case, the a large portion of the backshell is no longer within a fully separated wake. For this analysis, the claim is made that as the backshell pressure distribution becomes significantly influenced by attached flow and the wake ring-vortex structure breaks down at higher angles-of-attack, any lag in the base pressure has diminishing effect on the pitch damping of the vehicle. With the breakdown of the driving physical mechanism on the backshell, the forebody damping should dominate the vehicle damping behavior. The surface pressures on the backshell where the flow is attached will not lag like regions in separated wake flow. While the lag predictions of pitch damping will be made for all angle-of-attack solutions generated, this caveat should be noted when interpreting high angle-of-attack calculations.

\section{Results and Discussion}

\section{Backshell Geometry Effects}

$C_{m_{\dot{\alpha}}}$ predictions calculated using Equation 29 with the constant determined in Equation 31 are shown in Figures 5 and 6 . The local slope at each data point was determined using an overlapping parabola interpolation scheme to provide smooth and continuous interpolated values between data points. A finite difference derivative was then calculated at each data point. Figure 5 shows calculated damping values for both the Viking and MER capsules at Mach 3. These cases are plotted against MER ballistic range and Viking forced oscillation data. Note that the $C_{m_{\dot{\alpha}}}$ calculations were made at the center of gravity positions corresponding to the comparable data sources $\left(x_{c g} / d=0.27\right.$ and $x_{c g} / d=0.214$ for the MER and Viking cases). Overall, the agreement of the predictions with the experimental data is good. Both predictions indicate a region of decreased dynamic stability at lower angles-of-attack due to the lag of the backshell pressure distribution. In agreement with the experimental data, the lag calculations predict that the MER capsule is less dynamically stable than Viking at low angles. Quantitative conclusions about the higher angle-of-attack data are more difficult, but both predictions indicate increased dynamic stability at higher angles. Note that the ballistic range data is obtained by assuming a functional form and solving for the coefficients that model the behavior observed in the shadowgraphs. It is not surprising that the lag model shows local variation in the predicted damping curve that does not agree with the assumed form used in the 

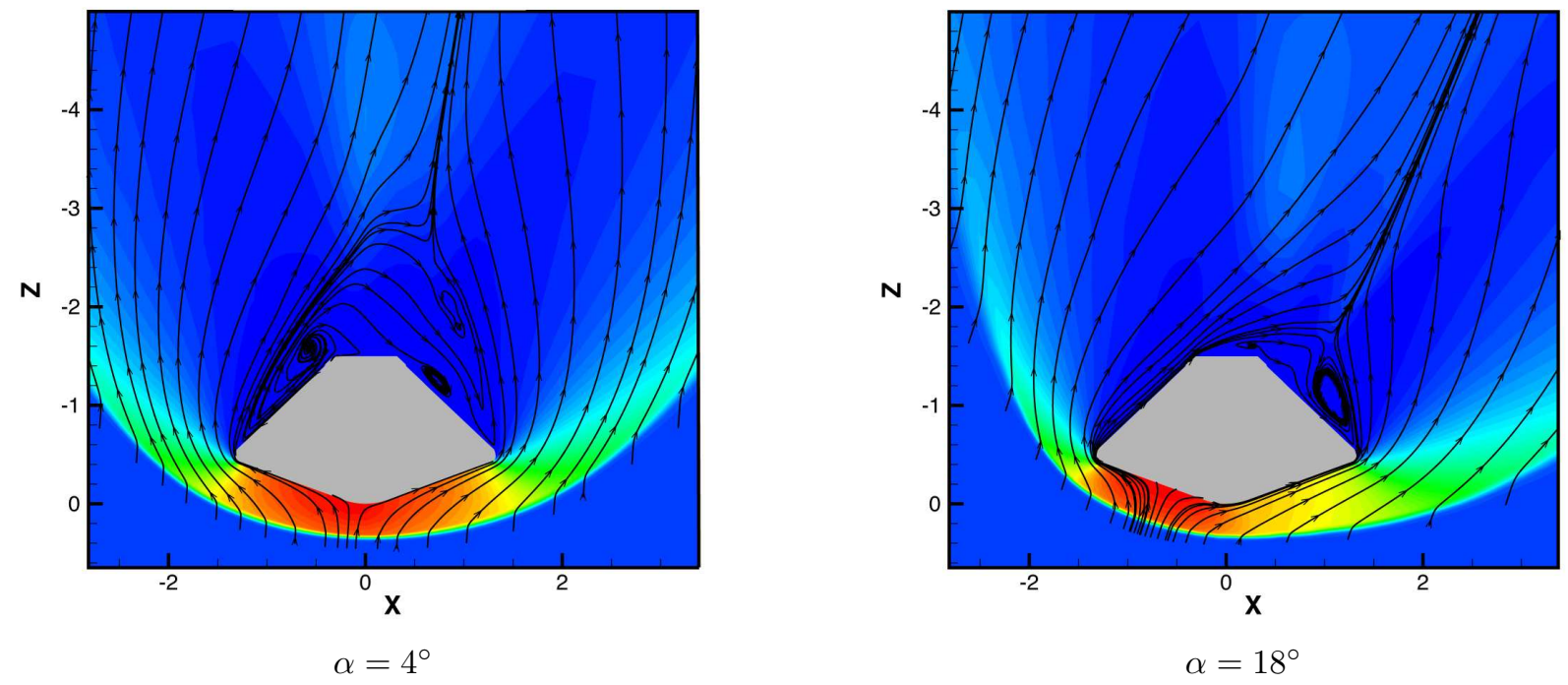

Figure 4. Mach = 3.0 LAURA solutions of the MER entry capsule

ballistic range data reduction. In fact, one possible use of this new method would be to help with initial guesses of the functional form to used in ballistic range analysis. The Viking lag predictions show trends that generally follow the same form as the forced oscillation curve although there are obvious discrepancies. The lag calculations predict that the peak dynamic instability is not at 0 degrees. This agrees with the forced oscillation data and runs counter to the assumptions used in the ballistic range data reduction efforts. Considering the crudeness with which the lag time was determined and the potential breakdown of the lag analysis at higher angles-of-attack, the predictions are remarkably similar to corresponding experimental data.

\section{Mach Number Sensitivity}

Figure 6 shows two MER cases at Mach $=2$ and 3 respectively, compared with ballistic range pitch damping curves. The lag predictions agree with the experimental data in one important area. At small angles, the lag calculations predict that the MER vehicle becomes more dynamically unstable with decreasing Mach number at lower angles-of-attack. The Mach 2 lag predictions show a much more significant peak instability near $5^{\circ}$ than the Mach 3 peak. However, in terms of functional form, the Mach 2 calculations differ from the ballistic range data considerably more than the Mach 3 data. The point of greatest dynamic instability is again away from $0^{\circ}$ and at larger angles there is an angle-of-attack range where the Mach 2 predictions indicate greater dynamic stability than the Mach 3 predictions. Again the method of selecting the lag time and the breakdown of the driving physics at high angles are likely sources of error. However, this prediction method's ability to capture low angle-of-attack Mach sensitivity to pitch damping is an important finding. Comparing the static stability of blunt body backshells may be used as an effective screening method. Estimates of lag time might be imprecise, but when comparing backshell concepts for a candidate vehicle, identifying relative differences is quite useful even if experiments are still required to quantify the damping characteristics of the shape ultimately selected. The data evaluated here indicates that meaningful comparisons are possible. Further, the functional forms of these shapes may help with ballistic range testing, providing a more educated starting point for data reduction analysis. Conversely, a CFD-based functional form might be used in the data reduction, and the phase angle that best replicated the ballistic range data points would be extracted. 


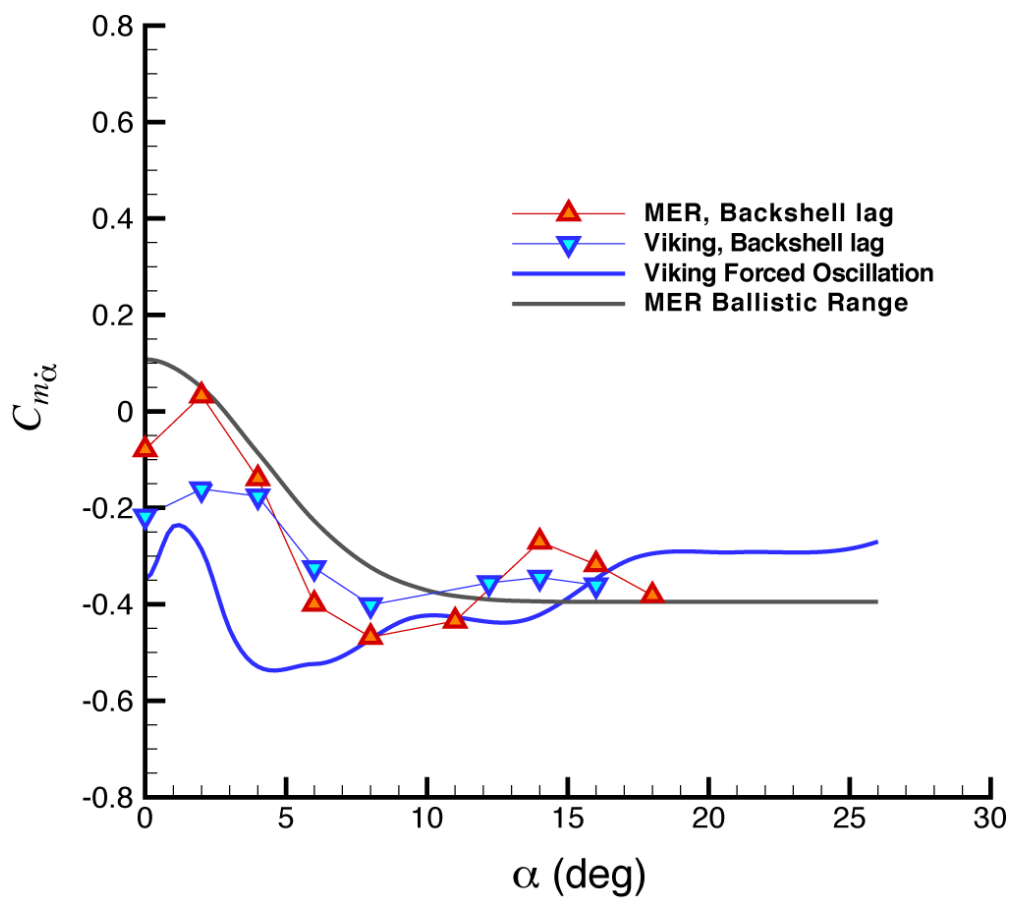

Figure 5. $C_{m_{\dot{\alpha}}}$ predictions for MER $\left(x_{c g} / D=0.27\right)$ and Viking $\left(x_{c g} / D=0.214\right)$ cases compared with ballistic range and forced oscillation data

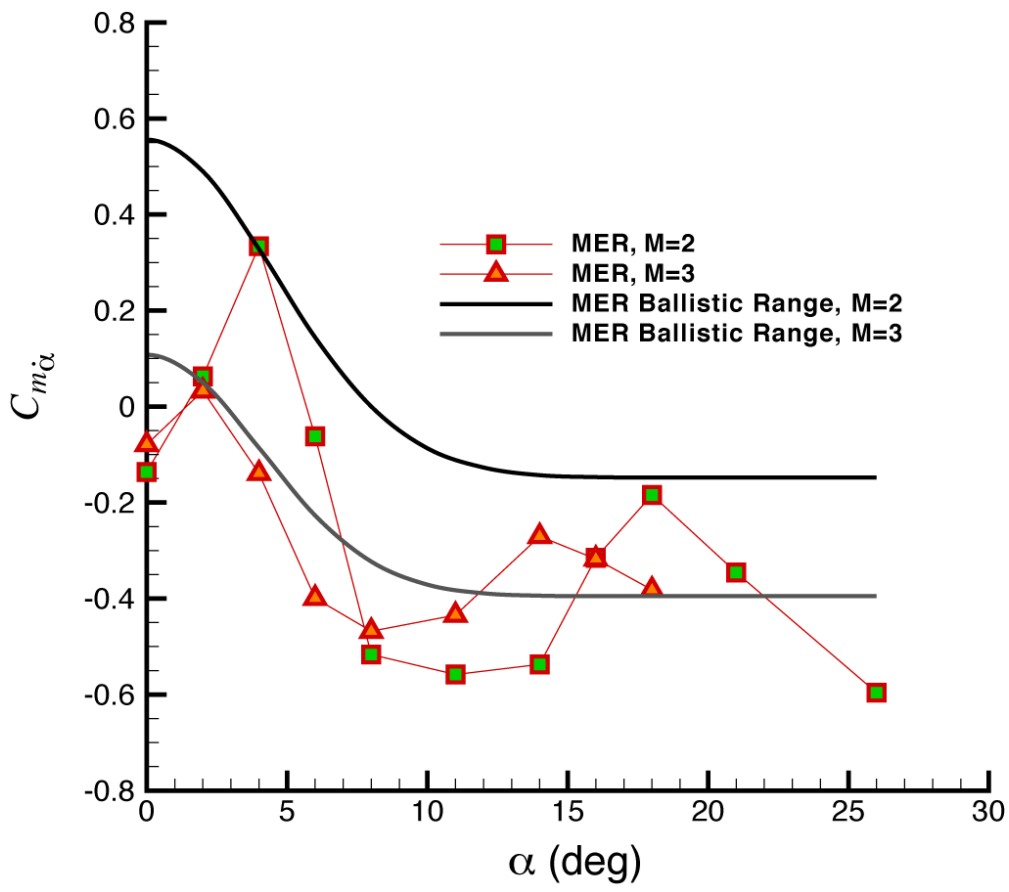

Figure 6. $C_{m_{\dot{\alpha}}}$ predictions for MER cases at Mach $=2$ and 3, compared with ballistic range data 


\section{Conclusions}

Lincarized analysis of the constant velocity moment equation has provided a simple method of predicting the dynamic stability of blunt entry capsules. This method relies only on static measurement or CFD calculation of the contribution to the pitching moment slope by the vehicle backshell. Comparison with MER and Viking experimental data has shown that this analysis can predict dynamic stability trends due to backshell geometry as well as Mach number. Dynamic stability characteristics were obtained for two blunt body geometrics that agreed well with damping characteristics measured experimentally. The only unknown parameter required to generate the lagged pitch-damping predictions was the phase angle by which the backshell moments lagged. The phase angle was taken directly from computational work by Teramoto et al. The comparisons with experiment data suggests this approach can be used in screening candidate backshell shapes and may assist in ballistic range data reduction.

A more rigorous method of determining the lag time, perhaps based on the momentum of the vortex structures in the wake flow, is a recommended next step. The static solutions indicate that the mechanisms assumed to drive the supersonic dynamic instabilities break down at higher angles of attack. Verifying that this breakdown occurs, and understanding that process could lead to improved results at higher angles-ofattack. An experimental investigation of the backshell pressure distributions and wake flowficlds behind these types of blunt body flows should be done to validate the pressure lag and underlying mechanisms identified with CFD by Teramoto et al. Flow visualization, surface pressure and moment measurements would all be helpful. Obtaining experimental data to better anchor this simple predictive model has the potential to more fully understand the physical mechanisms that cause blunt body dynamic instabilities, which to date have only been characterized with empirical data, supported with important, but limited, computational investigations.

\section{References}

${ }^{1}$ Steinberg, S., "Experimental Pitch Damping Derivatives for Candidate Viking Entry Configurations at Mach Numbers from 0.6 Through 3.0," TR-3709005, Martin Marictla Corporation, 1970.

${ }^{2}$ Stcinberg, S., Uselton, B., and Sicmers, P., "Viking Configuration Pitch Damping Derivatives as Influenced by Support Interference and 'lest 'Techmique at 'Transonic and Supersonic Speeds," AlAA 72-1012, September 1972.

${ }^{3}$ Owens, B. D. and lomek, D., " $18-\mathrm{CD}$ Subsonic-'Transonic Crew Module Dynamic Stability l'est in the NASA LaRC Transonic: Dynamics Tunnel," NASA/EG-CAP-07-99, February, 2009.

${ }^{4}$ Brown, J., Yates, L., Bogdanoff, D., Chapman, C., Loomis, M., and Tam, T., "Free Fligth Testing in Support of the Mars Smart Tander Aerodynamics Database," ATA 2002-1110, 2002.

${ }^{5}$ Tcramoto, S., Hiraki, K., and Fugii, K., "Numcrical Analysis of Dynamic Stability of a Recntry Capsulc at Transonic Speeds," AIAA Journal, Vol. 39, No. 4, April 2001, pp. 646-653.

${ }^{6}$ Schoenenberger, M. and Queen, L. M., "Limit Cycle Analysis Applied to the Oscillations of Decelerating Blunt-Body Entry Vehicles," NATO RT(--VP-AVT-152-006, 2008.

${ }^{7}$ Tobak, M. and Wehrend, W. R., "Stability Derivatives for Cones at Supersonic Speeds," NACA TN 3788, NACA, September 1956.

${ }^{8}$ Winchenbach, G. T., "Acrodynamic Testing in a Frec-Flight Spark Range," Tcch. Rep. WI-TR-1997-7006, Wright Taboratory, Armament Directorate, Weapon light Mechanics Division (WL/MNAV), Lglin Al'B, l'L, April 1997.

${ }^{9}$ Schoenenberger, M., llathaway, W., Yates, L., and Desai, P., "Ballistic Range 'lesting of the Mars Lxploration Rover Entry Capsule," AIAA 2005 0055, January 2005.

${ }^{10}$ Chapman, G. T., Mitcheltree, R. A., and Hathaway, W. H., "Transonic and Low Supersonic Static and Dyanmic Aerodynamic Characteristics of the Stardust Sample Return Capsule," $\Lambda \mathrm{I} \Lambda$ 99-1021, 1999.

${ }^{11}$ Flaherty, T. M., "Acrodynamics Data Book," TR- 3709011, Martin Marictla Corporation, 1972.

${ }^{12}$ Spencer, D. A., Blanchard, R. C., Braun, R. D., Kallemcyn, P. H., and Thurman, S. W., "Mars Pathfinder Fntry, Descent, and Landing Reconstruction," Journal of Spacecraft and Rockets, Vol. 36, No. 3, May-June 1999, pp. 357-366.

${ }^{13}$ Cheatwood, I. M. and Gnoffo, P. A., "Lser's Manual for the Langley Aerothermodynamic Upwind lielaxation Algorithm (LAURA)," NASA TM 4674, 1996.

${ }^{14}$ Schoenenberger, M., Cheatwood, F., and Desai, P., "Static Aerodynamics of the Mars Exploration Rover Entry Capsule," AI A $2005-0056$, January 2005. 